\title{
Celecoxib for Management of Refractory Back Pain Secondary to Vertebral Osteomyelitis: A Case Report
}

\author{
Michael Glicksman $^{\mathrm{a}, \mathrm{d}}$, Steven Jow ${ }^{\mathrm{b}}$, Laura Malmut ${ }^{\mathrm{c}}$
}

\begin{abstract}
Back pain is the most common symptom of vertebral osteomyelitis and can be difficult to manage. Pain may persist despite appropriate antibiotic medications and may be refractory to common analgesic treatments. We present a case of a 53-year-old man with acute onset severe low back pain. Clinical evaluation and diagnostic workup were consistent with L1 osteomyelitis. The patient continued to report pain following treatment with intravenous antibiotics and typical analgesic therapy. Opioids were discontinued and low-dose celecoxib was initiated with appreciable improvement in pain and activity tolerance. Celecoxib may be a good option and alternative to opioids in the pain management of this population.
\end{abstract}

Keywords: Pain management; Celecoxib; NSAIDs; Vertebral osteomyelitis; Low back pain; Non-opioid analgesics; Rehabilitation medicine

\section{Introduction}

Vertebral osteomyelitis (VO), additionally referred to as spondylodiscitis, is an infection of the vertebral body and intervertebral disc [1]. Infection may be caused by bacteria, fungi, tuberculosis, or other organisms [1]. It has been reported to constitute between $3 \%$ and $5 \%$ of all osteomyelitis cases and most frequently occurs in the lumbar spine [1, 2]. Infection most commonly spreads to the spine via hematogenous dis-

Manuscript submitted April 9, 2021, accepted April 23, 2021

Published online May 13, 2021

${ }^{a}$ Georgetown University School of Medicine, 3900 Reservoir Rd, NW, Washington, DC 20007, USA

bMedstar Georgetown University Hospital-National Rehabilitation Hospital, 102 Irving St NW, Washington, DC 20010, USA

${ }^{c}$ MedStar National Rehabilitation Hospital, 102 Irving St NW, Washington, DC 20010, USA

${ }^{\mathrm{d} C}$ Corresponding Author: Michael Glicksman, Georgetown University School of Medicine, 3900 Reservoir Rd, NW, Washington, DC 20007, USA.

Email:msg111@georgetown.edu

doi: https://doi.org/10.14740/jmc3699 semination, but direct inoculation from trauma or surgery and contiguous spread from adjacent tissues are also possible [3]. Risk factors include diabetes mellitus, renal failure, liver cirrhosis, autoimmune diseases, history of substance abuse and previous spinal surgeries $[2,4]$. VO occurs more commonly in males below 20 and between 50 and 70 years of age [4].

Common presenting symptoms include back pain (67$100 \%$ of cases) and fever (2-60\% of cases) [1]. Back pain is typically insidious, worse at night and can radiate to the chest or abdomen [1,4]. Pain that fails to resolve may become chronic and interfere with activities of daily living [2, $3]$. The profound impact of chronic low back pain as a disabling health condition is well-documented, with substantial negative impacts on quality of life and increased health care expenditures [2]. Despite these potential long-lasting consequences, there is a paucity of literature on pain and symptom control in VO.

Here, we detail the utilization of celecoxib for pain management in a 53-year-old man presenting with VO. The patient was informed that the data concerning the case would be submitted for publication and he provided consent.

\section{Case Report}

\section{Investigations}

A 53-year-old gentleman presented with acute onset, severe low back pain. Pain was localized to the lumbar spine and was 10 out of 10 in severity. The patient denied sensory changes and bladder or bowel incontinence. On evaluation, the patient was found to be febrile with focal tenderness over the lumbar spine and diffuse bilateral lower extremity weakness. Labs were notable for a normal white blood cell count with elevated C-reactive protein $(189 \mathrm{mg} / \mathrm{L})$ and erythrocyte sedimentation rate $(36 \mathrm{~mm} / \mathrm{h})$.

\section{Diagnosis}

Following admission to an acute care hospital, magnetic resonance imaging demonstrated abnormal bone marrow signal in the L1 vertebral body with abnormal soft tissue enhancement anterior to the L1 vertebral body concerning for either early 


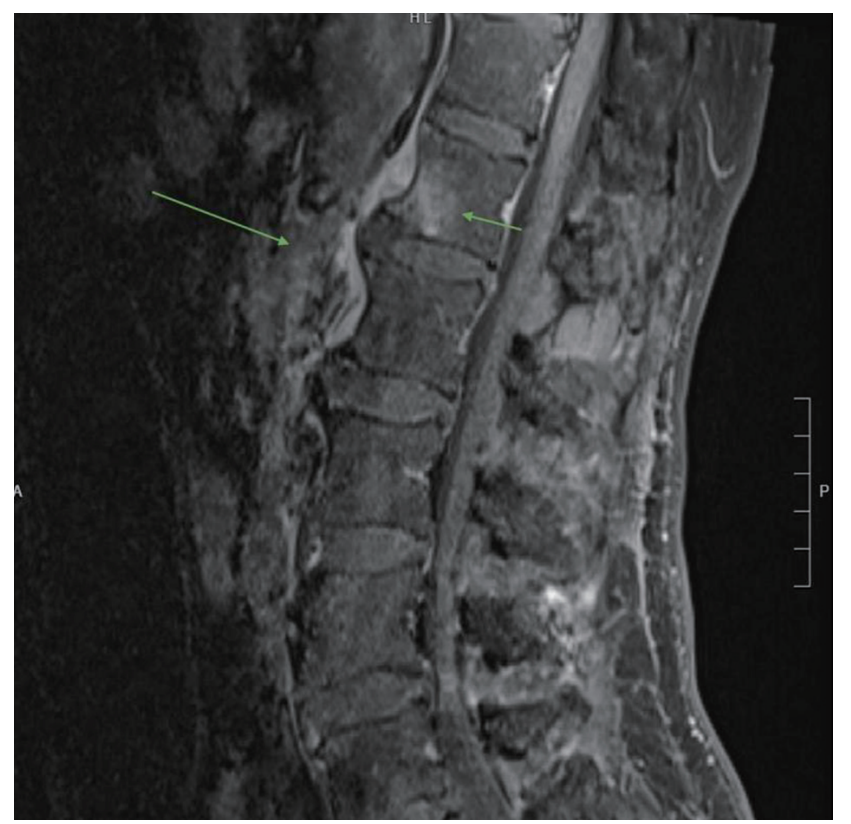

Figure 1. Sagittal T1-weighted image demonstrating increased signal intensity and enhancement of the anterior aspect of the inferior L1 vertebral body (short arrow) as well as abnormal soft tissue enhancement anterior and to the left of the L1 and L2 vertebral bodies (long arrow) suggestive of early osteomyelitis.

pyogenic or tuberculous osteomyelitis (Fig. 1). Blood bacterial, fungal and tuberculosis cultures, T-SPOT tuberculosis assay and bone biopsy were negative. Transthoracic echocardiogram revealed a mitral valve echodensity concerning for possible vegetation and severe mitral regurgitation. No vegetation was detected on transesophageal echocardiogram. Neurosurgery evaluated the patient and recommended conservative management.

\section{Treatment}

The patient completed 6 weeks of intravenous (IV) antibiotics with a final regimen of daptomycin (6 mg/kg IV every $24 \mathrm{~h}$ ) and ertapenem ( $1 \mathrm{~g}$ IV every $24 \mathrm{~h}$ ). Low back pain persisted despite antibiotic therapy and reduction in C-reactive protein (from 189 to $2.13 \mathrm{mg} / \mathrm{L}$ ) and erythrocyte sedimentation rate (from 36 to $13 \mathrm{~mm} / \mathrm{h}$ ). Initial pain medications included morphine, ketorolac and cyclobenzaprine. After 14 days of hospitalization, the patient was discharged to an inpatient rehabilitation facility on gabapentin, topical lidocaine patch, acetaminophen, baclofen and oxycodone.

The patient participated in a comprehensive inpatient rehabilitation program for 16 days. Endurance was poor, and he required physical assistance for transfers and ambulation. Activity tolerance and therapy participation were limited by severe pain. He never rated his pain less than a 5 out of 10 , and frequently endorsed pain up to 10 out of 10 on the numeric rating scale. Gabapentin, baclofen and oxycodone were discontinued. Celecoxib was initiated at a low dose of $100 \mathrm{mg}$ twice per day.

\section{Follow-up and outcomes}

Low back pain improved following initiation of low-dose celecoxib. Pain scores improved to 0 to 5 out of 10 and remained well controlled throughout the rehabilitation course. After starting celecoxib, he demonstrated increased participation in physical therapy and occupational therapy. At the time of discharge, the patient was independently ambulating with a walker and no longer required physical assistance for self-care. After discharge, the patient continued to use celecoxib on an as needed basis, and he endorsed, on average, 1 out of 10 pain at 6 months.

\section{Discussion}

This case demonstrates that low-dose celecoxib may effectively treat pain in patients with VO. Literature exploring the management of $\mathrm{VO}$ has focused on antibiotics, immobilization and surgical indications [3]. Even with more aggressive antibiotic regimens, back pain often persists for weeks to months due to gradual resolution of the infection [3]. Treatments directed at pain control typically involve oral and topical medications, modalities and physical therapy [3]. The most widely utilized oral medications in the treatment of pain related to spinal infections include opioids, tricyclic antidepressants and gabapentin [5]. Despite infrequent use in $\mathrm{VO}$, non-steroidal anti-inflammatory drugs (NSAIDs) can reduce both inflammatory and bone-related back pain in various conditions and may be beneficial for reducing pain in VO after effective antibiotics treatment [4].

Celecoxib is an NSAID that selectively inhibits cyclooxygenase-2 (COX-2) and can reduce osteoarthritic, rheumatological and post-operative pain [6]. Because celecoxib selectively targets COX-2, it poses a lower risk for gastrointestinal ulcers and bleeding that may result with other commonly used NSAIDs due to inhibition of cyclooxygenase-1 (COX-1) [7]. Pharmacokinetically, the high bioavailability, quick onset of action and propensity of celecoxib to preferentially distribute in inflamed tissue suggest that it may provide an effective pain management option in VO without many of the risks associated with opioids [6, 8]. A multicenter, randomized clinical trial comparing the efficacy of celecoxib to hydrocodone/acetaminophen in managing postoperative pain following ambulatory orthopedic surgeries demonstrated comparable levels of analgesia [8]. Selective COX-2 inhibitors have repeatedly shown significant benefit to patients undergoing spinal fusion surgeries as reflected by pain scores and opioid use [9]. Celecoxib's ability to reduce pain extends to other inflammatory and bonerelated conditions, such as multiple diaphyseal sclerosis, hypophosphatasia and chronic recurrent multifocal osteomyelitis [10-12]. This demonstrated efficacy of celecoxib in managing inflammatory bone pain suggests that it may be particularly beneficial in the management of pain related to VO. Literature investigating its efficacy is lacking; however, one animal study found that celecoxib significantly reduced pain behaviors in rats experimentally induced with osteomyelitis [13].

One potential explanation for the limited use of celecoxib in $\mathrm{VO}$ is a general concern that the non-steroidal anti-inflammatory properties may hinder bone healing [14]. More recent literature 
suggests that this is unlikely. A study by Reuben et al demonstrated no significant differences in the number of bone healing complications using celecoxib compared to those patients who did not receive NSAIDs [9]. A recent prospective cohort study confirmed these findings [15]. These conclusions suggest that celecoxib would not likely increase the risk of bone-related complications in VO managed either conservatively or operatively.

While the etiology of back pain in VO is not yet completely understood, the current literature suggests that prostaglandin E2 (PGE2) is fundamental [16]. A prior study demonstrated five- to thirty-fold increases in PGE2 production in infected bone compared to normal bone [16]. PGE2 may be the central prostanoid driving pain hypersensitivity due to its ability to regulate multiple nociceptive pathways involved in both peripheral and central sensitization [17]. Mechanical allodynia and thermal hyperalgesia may contribute to persistent pain in VO after the infection has resolved [13].

Celecoxib's anti-inflammatory and analgesic properties derive primarily from its ability to block the synthesis of prostanoids and downstream pro-inflammatory mediators including substance $\mathrm{P}$, bradykinin and histamine that activate nociceptors $[6,18]$. By these mechanisms, celecoxib may limit bone inflammation pain in VO [13]. In particular, celecoxib may preferentially target PGE2. One study showed up to $90 \%$ reduction of PGE2 with the use of celecoxib [18]. Thus, by targeting the specific pathways responsible for both acute and chronic back pain in VO, celecoxib may represent an efficacious option that may be used either alone or in combination with other pain management strategies.

Pain control is critical in the management of this disease. It is well documented that poorly controlled pain is associated with increased morbidity, reduced quality of life, impaired function, higher health care costs and increased duration of opioid use [19]. VO-mediated low back pain can persist for weeks to months and has been shown to contribute to impaired quality of life, long-term disability and increased healthcare costs [2]. From 1998 to 2013, hospital length of stay due to VO averaged 9.2 days [2]. Correcting for current costs, expenses secondary to VO in the USA alone would have surmounted to 1.3 billion dollars over 15 years [2].

As demonstrated by our case, uncontrolled pain hinders optimal engagement in rehabilitative therapies. Poor participation in inpatient physical and occupational therapies is associated with worse functional outcomes and longer hospital length of stays [20]. Adequate pain control may augment participation and improve metrics in walking ability, activity and quality of life following discharge [20].

In this case, the patient noticed a dramatic reduction in pain with low-dose celecoxib that was not previously achieved by opioids, gabapentinoids or another NSAID. Pain control spurred functional progression and was key to achieving near independence and home discharge. Future research should be directed toward the utilization of celecoxib for $\mathrm{VO}$ as a safe and effective option for pain relief after effective antibiotics treatment.

\section{Learning points}

Low back pain is the most common presenting symptom of
VO and may last for weeks to months following the initiation of antibiotic therapy. Celecoxib may be an effective treatment for acute and chronic pain in VO by targeting noxious mediators thought to be involved in osteomyelitic bone pain. Failure to treat persistent back pain secondary to VO can result in disability and increased health care expenditures. Celecoxib may be used as adjuvant therapy or as an alternative to commonly used agents (e.g., opioids, gabapentin) and may also be more appropriate than other NSAIDs in the treatment of VO-related bone pain.

\section{Acknowledgments}

None to declare.

\section{Financial Disclosure}

None to declare.

\section{Conflict of Interest}

None to declare.

\section{Informed Consent}

Informed consent was obtained prior to completing the case report.

\section{Author Contributions}

MG outlined the case report, conducted a comprehensive literature review and drafted the manuscript. He was also the submitting and corresponding author. SJ assisted with the design and editing of the case report, identified the MRI image and drafted the cover letter. LM was responsible for identifying this case as a candidate for a case report. She served as the point of contact for the patient in obtaining informed consent. She also edited the manuscript and guided the direction of the overall project.

\section{Data Availability}

The authors declare that data supporting the findings of this study are available within the article

\section{Abbreviations}

COX-1: cyclooxygenase-1; COX-2: cyclooxygenase-2; PGE2: prostaglandin E2; IV: intravenous; NSAIDs: non-steroidal anti-inflammatory drugs; VO: vertebral osteomyelitis 


\section{References}

1. Nickerson EK, Sinha R. Vertebral osteomyelitis in adults: an update. Br Med Bull. 2016;117(1):121-138.

2. Issa K, Diebo BG, Faloon M, Naziri Q, Pourtaheri S, Paulino CB, Emami A. The epidemiology of vertebral osteomyelitis in the United States from 1998 to 2013. Clin Spine Surg. 2018;31(2):E102-E108.

3. Gregori F, Grasso G, Iaiani G, Marotta N, Torregrossa F, Landi A. Treatment algorithm for spontaneous spinal infections: A review of the literature. J Craniovertebr Junction Spine. 2019;10(1):3-9.

4. Duarte RM, VaccaroAR. Spinal infection: state of the art and management algorithm. Eur Spine J. 2013;22(12):27872799.

5. Olsen ML, Havyer RD, Smith TJ, Swetz KM. Difficult cases of pain and nonpain symptoms in intractable spinal infections: a case series. Am J Hosp Palliat Care. 2012;29(6):493-496.

6. Gong L, Thorn CF, Bertagnolli MM, Grosser T, Altman RB, Klein TE. Celecoxib pathways: pharmacokinetics and pharmacodynamics. Pharmacogenet Genomics. 2012;22(4):310-318.

7. Dubois RW, Melmed GY, Henning JM, Bernal M. Risk of upper gastrointestinal injury and events in patients treated with cyclooxygenase (COX)-1/COX-2 Nonsteroidal Antiinflammatory Drugs (NSAIDs), COX-2 selective NSAIDs, and gastroprotective cotherapy: an appraisal of the literature. J Clin Rheumatol. 2004;10(4):178-189.

8. Gimbel JS, Brugger A, Zhao W, Verburg KM, Geis GS. Efficacy and tolerability of celecoxib versus hydrocodone/ acetaminophen in the treatment of pain after ambulatory orthopedic surgery in adults. Clin Ther. 2001;23(2):228241.

9. Reuben SS, Ablett D, Kaye R. High dose nonsteroidal anti-inflammatory drugs compromise spinal fusion. Can J Anaesth. 2005;52(5):506-512.

10. Cai Y, Lin H, Huang F, Zheng X, Huang Y, Zhang S. Im- aging features and differential diagnosis of multiple diaphyseal sclerosis: A case report and review of literature. Medicine (Baltimore). 2018;97(33):e11725.

11. Whyte MP, Wenkert D, McAlister WH, Mughal MZ, Freemont AJ, Whitehouse R, Baildam EM, et al. Chronic recurrent multifocal osteomyelitis mimicked in childhood hypophosphatasia. J Bone Miner Res. 2009;24(8):14931505.

12. Ferguson B, Gryfe D, Hsu W. Chronic recurrent multifocal osteomyelitis in a 13 year old female athlete: a case report. J Can Chiropr Assoc. 2013;57(4):334-340.

13. Yang CJ, Li Q, Wu GC, Wang YQ, Mao-Ying QL. A practical model of osteomyelitis-induced bone pain by intratibial injection of Staphylococcus aureus in rats. Neurosci Lett. 2012;513(2):198-203.

14. Gajraj NM. The effect of cyclooxygenase-2 inhibitors on bone healing. Reg Anesth Pain Med. 2003;28(5):456465.

15. Dinh A, Jean M, Bouchand F, Davido B, Descatha A, Duran $\mathrm{C}$, Gras $\mathrm{G}$, et al. Impact of anti-inflammatory drugs on pyogenic vertebral osteomyelitis: a prospective cohort study. Int J Rheumatol. 2016;2016:9345467.

16. Lazzarini L, De Lalla F, Mader JT. Long bone osteomyelitis. Curr Infect Dis Rep. 2002;4(5):439-445.

17. Chen L, Yang G, Grosser T. Prostanoids and inflammatory pain. Prostaglandins Other Lipid Mediat. 2013;104105:58-66.

18. Sinatra R. Role of COX-2 inhibitors in the evolution of acute pain management. J Pain Symptom Manage. 2002;24(1 Suppl):S18-27.

19. Gan TJ. Poorly controlled postoperative pain: prevalence, consequences, and prevention. J Pain Res. 2017;10:22872298.

20. Lenze EJ, Munin MC, Quear T, Dew MA, Rogers JC, Begley AE, Reynolds CF. Significance of poor patient participation in physical and occupational therapy for functional outcome and length of stay. Arch Phys Med Rehabil. 2004;85(10):1599-1601. 